\title{
Reduced Near-Resonant Vibrational Coupling at the Surfaces of Liquid Water and Ice
}

\author{
Wilbert J. Smit, ${ }^{* \dagger,} \S^{\circ}$ Jan Versluis, ${ }^{\dagger}$ Ellen H. G. Backus, ${ }^{\ddagger}{ }^{\ddagger}$ Mischa Bonn, ${ }^{\ddagger}{ }^{\ddagger}$ and Huib J. Bakker \\ ${ }^{\dagger}$ AMOLF, Science Park 104, 1098 XG Amsterdam, The Netherlands \\ ${ }^{\ddagger}$ Max Planck Institute for Polymer Research, Ackermannweg 10, 55128 Mainz, Germany \\ ${ }^{\S}$ PSL Research University, ESPCI Paris, UMR CBI 8231, 10 rue Vauquelin, 75005 Paris, France
}

\section{Supporting Information}

\begin{abstract}
We study the resonant interaction of the $\mathrm{OH}$ stretch vibrations of water molecules at the surfaces of liquid water and ice using heterodyne-detected sum-frequency generation (HD-SFG) spectroscopy. By studying different isotopic mixtures of $\mathrm{H}_{2} \mathrm{O}$ and $\mathrm{D}_{2} \mathrm{O}$, we vary the strength of the interaction, and we monitor the resulting effect on the HD-SFG spectrum of the $\mathrm{OH}$ stretch vibrations. We observe that the near-resonant coupling effects are weaker at the surface than in the bulk, for both water and ice, indicating that for both phases of water the $\mathrm{OH}$ vibrations are less strongly delocalized at the surface than in the bulk.
\end{abstract}

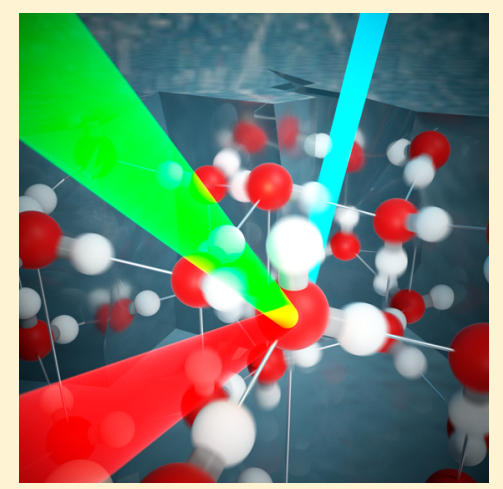

$\mathrm{W}$ ater and ice are remarkable substances owing to the presence of a spatially extended network of strong hydrogen bonds. This network is truncated at the surface, which gives rise to unique properties like an extremely high surface tension. The surface properties of water and ice govern a broad variety of biological and environmental processes. ${ }^{1-7}$ Therefore, a detailed understanding of the physical properties of the surface structure of water and ice at the molecular level is of broad scientific interest. Studying the surface properties of water and ice is challenging, as the surface region is typically very thin compared with the bulk. Few techniques are capable of probing the surfaces of water and ice with sufficient surface specificity.

Over the last three decades, sum-frequency generation (SFG) spectroscopy has emerged as an extremely useful tool to study the molecular structure of water surfaces. ${ }^{8-11}$ SFG is a second-order $\left(\chi^{(2)}\right)$ optical process that is dipole-forbidden in centrosymmetric media like bulk water but dipole-allowed at its surface. The method involves the frequency mixing of an infrared and a visible laser pulse, yielding a signal at the sum frequency. The SFG response is strongly enhanced when the infrared frequency matches a vibrational resonance, making it a selective probe for surface vibrations. The imaginary part of the second-order susceptibility $\chi^{(2)}$ can be interpreted as a surface absorption spectrum with the additional feature that, unlike a bulk absorption spectrum, $\operatorname{Im} \chi^{(2)}$ can be positive or negative. The sign of $\operatorname{Im} \chi^{(2)}$ reflects the net orientation of the transition dipole moment of the probed interfacial vibrational mode. In conventional SFG spectroscopy, the intensity of the sumfrequency signal is measured, yielding a signal that is proportional to the squared magnitude of the second-order susceptibility $\left(\left|\chi^{(2)}\right|^{2}\right)$. The real and imaginary parts of $\chi^{(2)}$ can be obtained using the recently developed heterodyne-detected SFG approach. ${ }^{12-16}$

The spectral response of the $\mathrm{OH}$ stretch vibrations of water depends on the strength of the hydrogen-bond interactions and on near-resonant intra- and intermolecular couplings. In neat $\mathrm{H}_{2} \mathrm{O}$, the stretching vibration of an $\mathrm{OH}$ group couples both to the other $\mathrm{OH}$ group located on the same $\mathrm{H}_{2} \mathrm{O}$ molecule (intramolecular coupling) and to $\mathrm{OH}$ oscillators located on other water molecules in the vicinity (intermolecular coupling). This coupling takes the form of a dipole-dipole coupling at longer distances (Förster energy transfer). At shorter distances higher electrical moments and even mechanical interactions may contribute. As a result of these couplings, the vibrational modes are delocalized over many $\mathrm{OH}$ oscillators, which leads to a broadening and red shift of the overall vibrational spectrum and precludes a direct connection between the hydrogen-bond strength and the $\mathrm{OH}$ vibrational frequency. ${ }^{17,18}$

We examine the effect of near-resonant coupling to the $\mathrm{OH}$ stretch vibrations at the surface of liquid water and ice by studying different isotopic mixtures of $\mathrm{H}_{2} \mathrm{O}$ and $\mathrm{D}_{2} \mathrm{O}$ using heterodyne-detected SFG spectroscopy. For isotopically dilute $\mathrm{HDO}$ in $\mathrm{D}_{2} \mathrm{O}$, the $\mathrm{OH}$ oscillator is decoupled from the surrounding water molecules containing predominantly OD oscillators, owing to the large frequency mismatch between the $\mathrm{OH}$ and $\mathrm{OD}$ oscillators. As a result, the $\operatorname{Im} \chi^{(2)}$ spectrum of

Received: December 20, 2017

Accepted: February 26, 2018

Published: February 26, 2018 
$\mathrm{HDO}: \mathrm{D}_{2} \mathrm{O}$ can be interpreted in terms of localized $\mathrm{OH}$ stretch oscillators.

A detailed description of the heterodyne-detected SFG setup and the data analysis is provided in the Supporting Information. In short, the SFG experiments in the $\mathrm{OH}$ stretch vibrational region are performed in an ssp-polarization configuration, which implies that the sum-frequency and visible beams are polarized perpendicular to the plane of incidence, whereas the infrared beam is polarized parallel to the plane of incidence. Interference of the sum-frequency signal generated at the sample surface with sum-frequency light generated from a gold surface (local oscillator) allows for the determination of the real and imaginary $x x z$ components of the second-order susceptibility $\chi^{(2)}$.

The $\operatorname{Im} \chi^{(2)}$ of the liquid water-air interface is presented in Figure 1 for different isotopic mixtures at room temperature

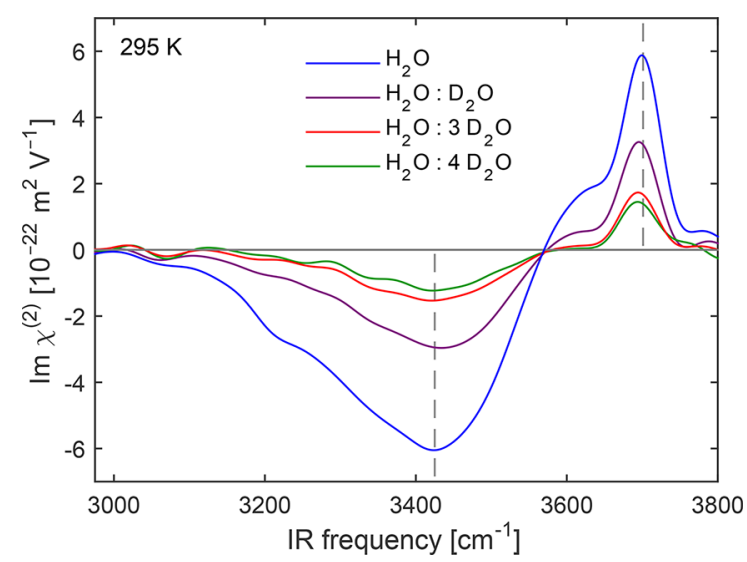

Figure 1. Imaginary part of the second-order susceptibility of the water-air interface at $295 \mathrm{~K}$ with various isotopic concentrations. The indicated $\mathrm{H}_{2} \mathrm{O}: n \mathrm{D}_{2} \mathrm{O}$ ratios quickly exchange to a mixture of $\mathrm{H}_{2} \mathrm{O}: \mathrm{HDO}: \mathrm{D}_{2} \mathrm{O} \simeq 1: 2 n: n^{2}{ }^{26}$ The maximum of the negative band and the position of the free $\mathrm{OH}$ mode of $\mathrm{H}_{2} \mathrm{O}$ are indicated by dashed lines.

(295 K). The signal scales with the number of chromophores and accordingly decreases with increasing isotopic dilution. The spectra show a main negative band centered at $\sim 3425 \mathrm{~cm}^{-1}$ and a positive band near $3700 \mathrm{~cm}^{-1}$. The $\operatorname{Im} \chi^{(2)}$ spectra of the liquid water-air interface shown in Figure 1 are very similar to other recently reported spectra. ${ }^{19-22}$ The observed $\operatorname{Im} \chi^{(2)}$ of liquid water is somewhat larger than previously reported values. ${ }^{19-21}$ All spectra show a zero-crossing near $\sim 3570 \mathrm{~cm}^{-1}$. The negative imaginary band below $3570 \mathrm{~cm}^{-1}$ corresponds to hydrogen-bonded $\mathrm{OH}$ groups. The negative value of $\operatorname{Im} \chi^{(2)}$ implies that the hydrogen-bonded $\mathrm{OH}$ groups have a net orientation of their hydrogen atoms pointing toward the bulk. The peak position is $\sim 3425 \mathrm{~cm}^{-1}$ and does not change with isotopic concentration.

The frequency of the free $\mathrm{OH}$ mode is affected by intramolecular coupling: We find that the position of the free $\mathrm{OH}$ mode shifts from $\sim 3701 \mathrm{~cm}^{-1}$ in pure $\mathrm{H}_{2} \mathrm{O}$ to $\sim 3692 \mathrm{~cm}^{-1}$ for diluted HDO by decomposing the $\operatorname{Im} \chi^{(2)}$ of the free $\mathrm{OH}$ mode by two Gaussian bands. The frequency of the free $\mathrm{OH}$ peak of $\mathrm{H}_{2} \mathrm{O}$ is $4 \mathrm{~cm}^{-1}$ higher than the value of $3697 \mathrm{~cm}^{-1}$ that was reported by Suzuki et al. ${ }^{23}$ This difference is within the experimental uncertainty of our experiment. In a previous study by Stiopkin et al. ${ }^{24}$ a shift of $\sim 17 \mathrm{~cm}^{-1}$ has been observed for the free OD mode. The observed shift of $\sim 9 \mathrm{~cm}^{-1}$ for the free
$\mathrm{OH}$ mode is smaller than for the free OD mode because the natural frequency is a factor of $\sim \sqrt{2}$ larger for the free $\mathrm{OH}$ mode, which increases the frequency mismatch with the other hydrogen-bonded $\mathrm{OH}$ mode on the same water molecule. Accordingly, the frequency shift resulting from the coupling is reduced for the free $\mathrm{OH}$ mode compared with the free $\mathrm{OD}$ mode. In addition, the intramolecular coupling is somewhat weaker in $\mathrm{H}_{2} \mathrm{O}$ than in $\mathrm{D}_{2} \mathrm{O}{ }^{24,25}$ The positive amplitude of the free $\mathrm{OH}$ mode in the $\operatorname{Im} \chi^{(2)}$ spectrum reflects its orientation with the hydrogen atom pointing away from the bulk. The Im $\chi^{(2)}$ spectrum of $\mathrm{H}_{2} \mathrm{O}$ shows a small positive offset above 3750 $\mathrm{cm}^{-1}$. The small positive offset is most likely due to the experimental error in the determined phase of $\chi^{(2)}$ of $\sim 10^{\circ}$.

The spectra shown in Figure 1 further reveal a shoulder at $\sim 3620 \mathrm{~cm}^{-1}$, of which the amplitude strongly decreases with isotopic dilution. For this band different explanations have been proposed. In one explanation, this response has been assigned to water molecules with two donor and one acceptor hydrogen bond. ${ }^{22,24,27}$ The $\mathrm{OH}$ groups of these molecules are oriented, on average, with their hydrogen atoms toward the bulk, and intramolecular coupling gives rise to an antisymmetric stretching mode with a positive $\operatorname{Im} \chi^{(2)}$ response in an ssppolarization configuration. ${ }^{28}$ In a more recent study, the shoulder at $\sim 3620 \mathrm{~cm}^{-1}$ has been assigned to a Fermi resonance between the free $\mathrm{OH}$ stretch $\left(\right.$ at $\sim 3700 \mathrm{~cm}^{-1}$ ) and a combination mode of a hydrogen-bonded $\mathrm{OH}$ stretch vibration $\left(\sim 3450 \mathrm{~cm}^{-1}\right)$ and a low-frequency intermolecular hydrogenbond vibration $\left(\sim 200 \mathrm{~cm}^{-1}\right){ }^{23}$ Both explanations would account for the observed dependence on isotope composition. With increasing isotopic dilution, the concentration of $\mathrm{H}_{2} \mathrm{O}$ decreases, and the intensity of a band resulting from either intramolecular coupling or a Fermi resonance involving closely spaced $\mathrm{OH}$ stretch vibrations will decrease accordingly.

To compare the shapes of the $\mathrm{H}_{2} \mathrm{O}$ and HDO: $\mathrm{D}_{2} \mathrm{O}$ spectra in more detail, we plot in Figure 2a the spectra normalized to the number of $\mathrm{OH}$ groups present. For $\mathrm{H}_{2} \mathrm{O}$, the negative $\mathrm{Im}$ $\chi^{(2)}$ band is broader (fwhm of $269 \mathrm{~cm}^{-1}$ ) than for HDO: $\mathrm{D}_{2} \mathrm{O}$ (fwhm of $208 \mathrm{~cm}^{-1}$ ). In addition, the first spectral moment of
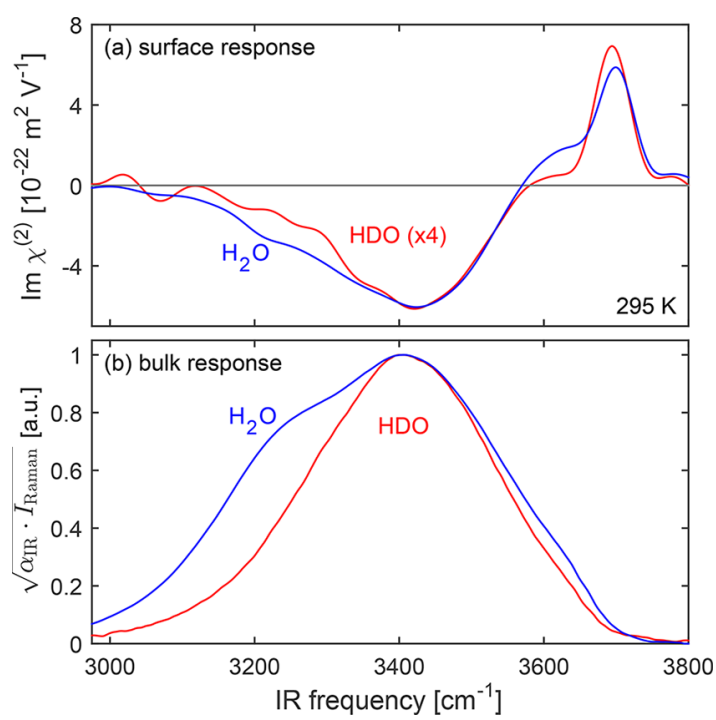

Figure 2. (a) Imaginary part of the second-order susceptibility of the surface of $\mathrm{H}_{2} \mathrm{O}$ and $\mathrm{H}_{2} \mathrm{O}: 3 \mathrm{D}_{2} \mathrm{O}$ at $295 \mathrm{~K}$. (b) Square oot of the product of infrared and Raman spectra $\left(\sqrt{\alpha_{\mathrm{IR}} \cdot I_{\text {Raman }}}\right)$ of $\mathrm{H}_{2} \mathrm{O}$ and $\mathrm{H}_{2} \mathrm{O}: 3 \mathrm{D}_{2} \mathrm{O}$ at $295 \mathrm{~K}$. 
this band is red-shifted by $\sim 19 \mathrm{~cm}^{-1}$. These differences result from the resonant coupling of the $\mathrm{OH}$ oscillators for pure $\mathrm{H}_{2} \mathrm{O}$; for $\mathrm{HDO}: \mathrm{D}_{2} \mathrm{O}$ this effect vanishes. ${ }^{22,29}$

The broadening and red shift of the spectrum can be explained from the near-resonant interactions between the $\mathrm{OH}$ stretch vibrations. The near-resonant coupling of the $\mathrm{OH}$ stretch vibrations in pure $\mathrm{H}_{2} \mathrm{O}$ leads to the formation of a band of delocalized exciton $\mathrm{OH}$ vibrations that is broader than the inhomogeneous distribution of uncoupled $\mathrm{OH}$-stretch vibrational frequencies, thus explaining the larger width of the negative $\operatorname{Im} \chi^{(2)}$ band of $\mathrm{H}_{2} \mathrm{O}$ compared with dilute HDO: $\mathrm{D}_{2} \mathrm{O}$. The $\mathrm{OH}$ vibrations are in-phase for the lower-frequency exciton modes in this band and out-of-phase for the higher frequency exciton modes in this band. As a result, the low-frequency exciton modes have a larger absorption cross-section than the high-frequency exciton modes, leading to a red shift of the first spectral moment. Recent molecular dynamics simulations confirmed that the near-resonant coupling of the $\mathrm{OH}$ stretch vibrations leads to a broadening and red shift of the $\mathrm{OH}$ stretch $\operatorname{Im} \chi^{(2)}$ spectrum. $^{22}$ The calculated results are in qualitative but not quantitative agreement with the experimental findings; for example, the calculated red shift of the spectrum is larger than is experimentally observed.

To compare the surface $\operatorname{Im} \chi^{(2)}$ spectra with the bulk response, one should note that the SFG response is the product of the infrared transition dipole moment and the Raman polarizability. We therefore plot in Figure $2 \mathrm{~b}$ the constructed $\sqrt{\alpha_{\text {IR }} \cdot I_{\text {Raman }}}$ spectra of $\mathrm{H}_{2} \mathrm{O}$ and isotopically diluted water (HDO: $\mathrm{D}_{2} \mathrm{O}$ ) (with $\alpha_{\mathrm{IR}}$ and $I_{\text {Raman }}$ as the bulk infrared and Raman signals, respectively; see the Supporting Information for experimental details). For dilute $\mathrm{HDO}: \mathrm{D}_{2} \mathrm{O}$, the surface and bulk spectra show a quite similar width and shape, and the maximum of the $\operatorname{Im} \chi^{(2)}$ spectrum is blue-shifted by only $\sim 20$ $\mathrm{cm}^{-1}$ with respect to the maximum of the bulk spectrum. These observations show that the hydrogen bonds at the surface are quite comparable in strength to the hydrogen bonds in the bulk, in line with the observation of a remarkably fast onset of a bulk-phase hydrogen-bond structure when entering the surface of water. $^{11,24,30,31}$

The $\mathrm{H}_{2} \mathrm{O}$ bulk spectrum is strongly affected by intra- and intermolecular coupling, ${ }^{17}$ leading to a substantial broadening from 306 to $413 \mathrm{~cm}^{-1}$ (fwhm) and a red shift of the first spectral moment by $\sim 34 \mathrm{~cm}^{-1}$ with respect to the first spectral moment of the HDO: $\mathrm{D}_{2} \mathrm{O}$ spectrum. The shift of the first spectral moment due to the near-resonant coupling of the $\mathrm{OH}$ stretch vibrations is thus about two times larger for the bulk spectrum than for the surface spectrum. This finding indicates that the degree of vibrational delocalization is smaller at the surface of liquid $\mathrm{H}_{2} \mathrm{O}$ than in the bulk of liquid $\mathrm{H}_{2} \mathrm{O}$. This observation agrees with the results of molecular dynamics simulations performed by Auer and Skinner ${ }^{17,32}$ and of timeresolved studies of interfacial vibrational energy transfer. ${ }^{30,31}$ The former have revealed the vibrational eigenstates to be delocalized over up to $12 \mathrm{OH}$ groups in the bulk and over 1-6 $\mathrm{OH}$ groups close to the surface. ${ }^{17,32}$ This decrease in vibrational delocalization at the surface is probably largely a consequence of the difference in local geometry: At the surface an $\mathrm{OH}$ oscillator is surrounded by fewer $\mathrm{OH}$ groups than in the bulk. Experimental studies on vibrational relaxation and resonant energy transfer of the surface and the bulk of liquid $\mathrm{H}_{2} \mathrm{O}$ have shown that the rate of resonant vibrational energy transfer among $\mathrm{OH}$ stretch vibrations is approximately halved at the surface of liquid $\mathrm{H}_{2} \mathrm{O}$ with respect to the bulk, owing to the lower number of potential energy acceptors at the surface. ${ }^{30,31}$

Next, we compare the surface of water with the surface of ice. The growth and processing of single-crystal ice samples are described in ref 33. In Figure 3a we show the $\operatorname{Im} \chi^{(2)}$ spectrum
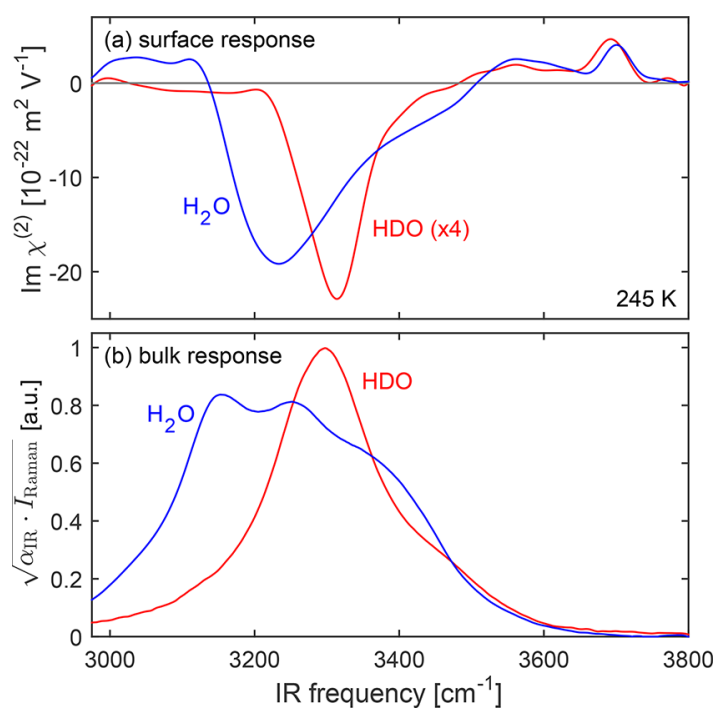

Figure 3. (a) Imaginary part of the second-order susceptibility of the basal surface of $\mathrm{H}_{2} \mathrm{O}$ ice and $\mathrm{H}_{2} \mathrm{O}: 3 \mathrm{D}_{2} \mathrm{O}$ ice at $245 \mathrm{~K}$. (b) Square-root product of the infrared and Raman spectra $\left(\sqrt{\alpha_{\mathrm{IR}} \cdot I_{\mathrm{Raman}}}\right)$ of $\mathrm{H}_{2} \mathrm{O}$ ice and $\mathrm{H}_{2} \mathrm{O}: 3 \mathrm{D}_{2} \mathrm{O}$ ice at $245 \mathrm{~K}$.

of the basal faces of $\mathrm{H}_{2} \mathrm{O}$ and $\mathrm{H}_{2} \mathrm{O}: 3 \mathrm{D}_{2} \mathrm{O}$ ice single crystals at $245 \mathrm{~K}$. Recently, the SFG spectrum of $\mathrm{H}_{2} \mathrm{O}$ ice at ca. $130 \mathrm{~K}$ was reported by Nojima et al. ${ }^{34}$ This SFG spectrum contains a positive band at $3100 \mathrm{~cm}^{-1}$. This band is not the same as the band that dominates the SFG spectrum at $245 \mathrm{~K}$ shown in Figure 3a. At temperatures below $200 \mathrm{~K}$, the SFG spectrum of ice contains several $\mathrm{OH}$-stretch bands, including a band at 3100 $\mathrm{cm}^{-1}$ for which we observed a dispersive line shape in the imaginary part, ${ }^{33}$ that is, $\pi / 2$ phase shifted in comparison with the band reported in ref 34 . The band at $3100 \mathrm{~cm}^{-1}$ likely represents the response of water molecules deeper buried in the ice crystal. At temperatures above $200 \mathrm{~K}$, this bulk band becomes small, ${ }^{33}$ and the SFG spectrum is dominated by the band near $3200 \mathrm{~cm}^{-1}$ that has an absorptive imaginary response at all temperatures and that represents the response of water molecules at the surface. Otsuki et al. ${ }^{35}$ measured the spectrum of HDO: $\mathrm{D}_{2} \mathrm{O}$ ice at $120 \mathrm{~K}$. This spectrum has a different shape from the spectrum shown here, which can be explained from the fact that the previous study was performed at a much lower temperature and for a different polarization combination. It has been shown that the SFG spectrum of ice strongly depends on temperature. $^{33,36}$

Above $3500 \mathrm{~cm}^{-1}$, the $\operatorname{Im} \chi^{(2)}$ spectrum is positive and contains a resonance at $\sim 3700 \mathrm{~cm}^{-1}$, corresponding to nonhydrogen-bonded free $\mathrm{OH}$ groups sticking out of the ice surface, and a broad positive response between 3500 and 3600 $\mathrm{cm}^{-1}$. The latter response has been assigned to the antisymmetric stretch vibration of $\mathrm{H}_{2} \mathrm{O}$ molecules near the surface. ${ }^{33}$ This signal decreases less upon isotopic dilution than expected, probably because of the narrowing of the strong negative signal between 3150 and $3450 \mathrm{~cm}^{-1}$. The position of the free $\mathrm{OH}$ is $\sim 8 \mathrm{~cm}^{-1}$ higher for $\mathrm{H}_{2} \mathrm{O}$ ice than for $\mathrm{HDO}: \mathrm{D}_{2} \mathrm{O}$ ice, which can be explained from the intramolecular coupling of 
$\mathrm{H}_{2} \mathrm{O}$, similar to what is observed for the surface of liquid $\mathrm{H}_{2} \mathrm{O}$ (see above). The observed frequency shift for ice is slightly smaller than the $\sim 9 \mathrm{~cm}^{-1}$ shift observed for liquid water, which can be well explained from the larger frequency difference between the free $\mathrm{OH}$ oscillator and the other hydrogen-bonded $\mathrm{OH}$ group located on the same $\mathrm{H}_{2} \mathrm{O}$ molecule for $\mathrm{H}_{2} \mathrm{O}$ ice.

The spectral shapes of the $\operatorname{Im} \chi^{(2)}$ response of $\mathrm{H}_{2} \mathrm{O}$ and HDO: $\mathrm{D}_{2} \mathrm{O}$ ice are very different below $3500 \mathrm{~cm}^{-1}$ (Figure 3a). The negative imaginary band of the $\mathrm{H}_{2} \mathrm{O}$ spectrum is dominated by a band at $3230 \mathrm{~cm}^{-1}$. A small shoulder band is located near $\sim 3450 \mathrm{~cm}^{-1} .37$ The Im $\chi^{(2)}$ spectrum of $\mathrm{HDO}: \mathrm{D}_{2} \mathrm{O}$ ice is dominated by a single negative imaginary band at $\sim 3310 \mathrm{~cm}^{-1}$. For $\mathrm{H}_{2} \mathrm{O}$ ice the negative $\operatorname{Im} \chi^{(2)}$ is broader (fwhm of $168 \mathrm{~cm}^{-1}$ ) than for HDO: $\mathrm{D}_{2} \mathrm{O}$ ice (fwhm of $\left.91 \mathrm{~cm}^{-1}\right)$. In addition, the first spectral moment of this band is red-shifted by $\sim 33 \mathrm{~cm}^{-1}$ compared with $\mathrm{HDO}: \mathrm{D}_{2} \mathrm{O}$. The effect of near-resonant coupling of the $\mathrm{OH}$ stretch vibrations is thus stronger at the surface of $\mathrm{H}_{2} \mathrm{O}$ ice than at the surface of $\mathrm{H}_{2} \mathrm{O}$ liquid water, for which we observed a red shift of $\sim 19 \mathrm{~cm}^{-1}$. Recent molecular dynamics simulations by Ishiyama and Morita also found the $\mathrm{OH}$ stretch vibrations at the surface of $\mathrm{H}_{2} \mathrm{O}$ ice to be strongly delocalized. ${ }^{38}$ However, the shapes of the calculated spectra of both $\mathrm{H}_{2} \mathrm{O}$ and $\mathrm{HDO}: \mathrm{D}_{2} \mathrm{O}$ are not in agreement with the measured spectra.

The stronger coupling of the $\mathrm{OH}$ stretch vibrations at the surface of ice can be explained from the fact that ice has a narrower distribution of uncoupled $\mathrm{OH}$ stretch vibrational frequencies than liquid water. Because of this narrower distribution, the resonant dipole-dipole interactions result in a stronger mixing of the $\mathrm{OH}$ vibrations in ice than in water, both in bulk and at the surfaces. In addition, the $\mathrm{OH}$ stretch transition dipole moment is $20 \%$ larger for ice than for liquid water, ${ }^{39}$ leading to a stronger intra- and intermolecular dipoledipole coupling and thus to a larger degree of delocalization of the $\mathrm{OH}$ stretch vibrations for $\mathrm{H}_{2} \mathrm{O}$ ice than for $\mathrm{H}_{2} \mathrm{O}$ liquid water.

In Figure $3 \mathrm{~b}$ we present, for comparison, bulk $\sqrt{\alpha_{\mathrm{IR}} \cdot I_{\text {Raman }}}$ spectra of $\mathrm{H}_{2} \mathrm{O}$ and $\mathrm{H}_{2} \mathrm{O}: 3 \mathrm{D}_{2} \mathrm{O}$ ice at $245 \mathrm{~K}$. The isotopically diluted spectrum is dominated by a single band at $\sim 3300 \mathrm{~cm}^{-1}$. This band is at a lower frequency for HDO: $\mathrm{D}_{2} \mathrm{O}$ ice than for liquid HDO: $\mathrm{D}_{2} \mathrm{O}$, reflecting the larger average strength of the hydrogen bonds in ice than in the liquid. The band is also narrower for HDO: $\mathrm{D}_{2} \mathrm{O}$ ice than for liquid $\mathrm{HDO}: \mathrm{D}_{2} \mathrm{O}$, which indicates that the distribution of hydrogen-bond strengths is narrower in ice than in liquid water. For HDO: $\mathrm{D}_{2} \mathrm{O}$ ice, the bulk resonance frequency is close to the maximum of the sumfrequency spectrum, red-shifted by only $\sim 10 \mathrm{~cm}^{-1}$. Hence, the hydrogen bonds have a similar strength at the surface of ice surface as in the bulk of ice, in line with the observations for liquid water.

The bulk spectra of HDO: $\mathrm{D}_{2} \mathrm{O}$ and $\mathrm{H}_{2} \mathrm{O}$ ice strongly differ, which can be explained from the strong effects of near-resonant coupling and the resulting delocalization of the $\mathrm{OH}$ oscillators in $\mathrm{H}_{2} \mathrm{O}$ ice. ${ }^{25,40}$ The $\mathrm{H}_{2} \mathrm{O}$ bulk spectrum is broader (fwhm of $357 \mathrm{~cm}^{-1}$ ) than the bulk spectrum of HDO: $\mathrm{D}_{2} \mathrm{O}$ (fwhm of 170 $\mathrm{cm}^{-1}$ ) and has a first spectral moment that is red-shifted by $\sim 63 \mathrm{~cm}^{-1}$ with respect to the first spectral moment of the $\mathrm{HDO}: \mathrm{D}_{2} \mathrm{O}$ bulk spectrum. This shift is about two times larger than for the surface of $\mathrm{H}_{2} \mathrm{O}$ ice. Clearly, the near-resonant coupling of the $\mathrm{OH}$ stretch vibrations results in much stronger spectral broadening and stronger spectra red shift for bulk $\mathrm{H}_{2} \mathrm{O}$ ice than for the surface of $\mathrm{H}_{2} \mathrm{O}$ ice.
We observe the relative decrease in spectral red shift going from the bulk to the surface to be quite similar for water and ice. For both phases of water we find a decrease in the red shift of the first spectral moment by a factor of 1.9: from 34 to 19 $\mathrm{cm}^{-1}$ for liquid water and from 63 to $33 \mathrm{~cm}^{-1}$ for ice. This similarity in the decrease in the red shift suggests that the reduction of the near-resonant coupling of the $\mathrm{OH}$ stretch vibrations at the surface is mainly a geometrical effect. For both water and ice, the density of $\mathrm{OH}$ oscillators decreases by a factor of $\sim 2$ going from the bulk to the surface, with the result that the vibrational exciton modes at the surface involve a smaller number of local $\mathrm{OH}$ oscillators than the vibrational exciton modes in the bulk. This smaller degree of delocalization at the surface reduces the broadening and red shift of the surface spectrum.

In summary, we studied the effects of near-resonant intraand intermolecular interactions at the surfaces of liquid water and ice using heterodyne-detected SFG experiments. For both liquid water at $295 \mathrm{~K}$ and ice at $245 \mathrm{~K}$, we observed a broadening and a red shift of the first spectral moment of the hydrogen-bonded $\operatorname{Im} \chi^{(2)}$ spectrum of neat $\mathrm{H}_{2} \mathrm{O}$ with respect to isotopically diluted $\left(\mathrm{HDO}: \mathrm{D}_{2} \mathrm{O}\right)$ water. The red shift is stronger for ice than for water, which indicates that the $\mathrm{OH}$ vibrations are more strongly delocalized at the ice surface than at the water surface. By comparing the $\operatorname{Im} \chi^{(2)}$ spectra with the bulk response, we conclude that the near-resonant coupling effects are weaker at the surface than in the bulk, for both water and ice, indicating that for both phases of water the $\mathrm{OH}$ vibrations are less strongly delocalized at the surface than in the bulk.

\section{ASSOCIATED CONTENT}

\section{S Supporting Information}

The Supporting Information is available free of charge on the ACS Publications website at DOI: 10.1021/acs.jpclett.7b03359.

Description of the sample preparation, experimental setup, and analysis. (PDF)

\section{AUTHOR INFORMATION}

\section{Corresponding Author}

*E-mail: smit@amolf.nl.

\section{ORCID $\odot$}

Wilbert J. Smit: 0000-0002-0543-7258

Ellen H. G. Backus: 0000-0002-6202-0280

Mischa Bonn: 0000-0001-6851-8453

\section{Notes}

The authors declare no competing financial interest.

\section{ACKNOWLEDGMENTS}

This work is part of the research programme of The Netherlands Organisation for Scientific Research (NWO). We thank Hinco Schoenmaker and Dion Ursem for technical support and Walter Scholdei for measuring the Raman spectra.

\section{REFERENCES}

(1) Richmond, G. L. Molecular Bonding and Interactions at Aqueous Surfaces as Probed by Vibrational Sum Frequency Spectroscopy. Chem. Rev. 2002, 102, 2693-2724.

(2) Dash, J. G.; Fu, H.; Wettlaufer, J. S. The premelting of ice and its environmental consequences. Rep. Prog. Phys. 1995, 58, 115. 
(3) Dash, J. G.; Rempel, A. W.; Wettlaufer, J. S. The physics of premelted ice and its geophysical consequences. Rev. Mod. Phys. 2006, 78, 695-741.

(4) Molina, M. J.; Tso, T.-L.; Molina, L. T.; Wang, F. C.-Y. Antarctic Stratospheric Chemistry of Chlorine Nitrate, Hydrogen Chloride, and Ice: Release of Active Chlorine. Science 1987, 238, 1253-1257.

(5) Bolton, K.; Pettersson, J. B. C. Ice-Catalyzed Ionization of Hydrochloric Acid. J. Am. Chem. Soc. 2001, 123, 7360-7363.

(6) Bartels-Rausch, T.; Jacobi, H.-W.; Kahan, T. F.; Thomas, J. L.; Thomson, E. S.; Abbatt, J. P. D.; Ammann, M.; Blackford, J. R.; Bluhm, H.; Boxe, C.; et al. A review of air-ice chemical and physical interactions (AICI): liquids, quasi-liquids, and solids in snow. Atmos. Chem. Phys. 2014, 14, 1587-1633.

(7) Björneholm, O.; Hansen, M. H.; Hodgson, A.; Liu, L.-M.; Limmer, D. T.; Michaelides, A.; Pedevilla, P.; Rossmeisl, J.; Shen, H.; Tocci, G.; et al. Water at Interfaces. Chem. Rev. 2016, 116, 7698-7726.

(8) Shen, Y. R.; Ostroverkhov, V. Sum-Frequency Vibrational Spectroscopy on Water Interfaces: Polar Orientation of Water Molecules at Interfaces. Chem. Rev. 2006, 106, 1140-1154.

(9) Du, Q.; Superfine, R.; Freysz, E.; Shen, Y. R. Vibrational spectroscopy of water at the vapor/water interface. Phys. Rev. Lett. 1993, 70, 2313-2316.

(10) Shen, Y. R. Phase-Sensitive Sum-Frequency Spectroscopy. Annu. Rev. Phys. Chem. 2013, 64, 129-150.

(11) Bonn, M.; Nagata, Y.; Backus, E. H. G. Molecular Structure and Dynamics of Water at the Water-Air Interface Studied with SurfaceSpecific Vibrational Spectroscopy. Angew. Chem., Int. Ed. 2015, 54, $5560-5576$

(12) Ji, N.; Ostroverkhov, V.; Chen, C.-Y.; Shen, Y.-R. PhaseSensitive Sum-Frequency Vibrational Spectroscopy and Its Application to Studies of Interfacial Alkyl Chains. J. Am. Chem. Soc. 2007, 129, 10056-10057.

(13) Ji, N.; Ostroverkhov, V.; Tian, C. S.; Shen, Y. R. Characterization of Vibrational Resonances of Water-Vapor Interfaces by PhaseSensitive Sum-Frequency Spectroscopy. Phys. Rev. Lett. 2008, 100, 096102.

(14) Stiopkin, I. V.; Jayathilake, H. D.; Bordenyuk, A. N.; Benderskii, A. V. Heterodyne-Detected Vibrational Sum Frequency Generation Spectroscopy. J. Am. Chem. Soc. 2008, 130, 2271-2275.

(15) Yamaguchi, S.; Tahara, T. Heterodyne-detected electronic sum frequency generation: "Up" versus "down" alignment of interfacial molecules. J. Chem. Phys. 2008, 129, 101102.

(16) Nihonyanagi, S.; Yamaguchi, S.; Tahara, T. Direct evidence for orientational flip-flop of water molecules at charged interfaces: A heterodyne-detected vibrational sum frequency generation study. J. Chem. Phys. 2009, 130, 204704.

(17) Auer, B. M.; Skinner, J. L. IR and Raman spectra of liquid water: Theory and interpretation. J. Chem. Phys. 2008, 128, 224511.

(18) Auer, B. M.; Skinner, J. L. Vibrational sum-frequency spectroscopy of the liquid/vapor interface for dilute HOD in $\mathrm{D}_{2} \mathrm{O}$. J. Chem. Phys. 2008, 129, 214705.

(19) Yamaguchi, S. Development of single-channel heterodynedetected sum frequency generation spectroscopy and its application to the water/vapor interface. J. Chem. Phys. 2015, 143, 034202.

(20) Nihonyanagi, S.; Kusaka, R.; Inoue, K.-I.; Adhikari, A.; Yamaguchi, S.; Tahara, T. Accurate determination of complex $\chi^{(2)}$ spectrum of the air/water interface. J. Chem. Phys. 2015, 143, 124707.

(21) Sun, S.; Liang, R.; Xu, X.; Zhu, H.; Shen, Y. R.; Tian, C. Phase reference in phase-sensitive sum-frequency vibrational spectroscopy. J. Chem. Phys. 2016, 144, 244711.

(22) Schaefer, J.; Backus, E. H. G.; Nagata, Y.; Bonn, M. Both Interand Intramolecular Coupling of $\mathrm{O}-\mathrm{H}$ Groups Determine the Vibrational Response of the Water/Air Interface. J. Phys. Chem. Lett. 2016, 7, 4591-4595.

(23) Suzuki, Y.; Nojima, Y.; Yamaguchi, S. Vibrational Coupling at the Topmost Surface of Water Revealed by Heterodyne-Detected Sum Frequency Generation Spectroscopy. J. Phys. Chem. Lett. 2017, 8, 1396-1401.
(24) Stiopkin, I. V.; Weeraman, C.; Pieniazek, P. A.; Shalhout, F. Y.; Skinner, J. L.; Benderskii, A. V. Hydrogen bonding at the water surface revealed by isotopic dilution spectroscopy. Nature 2011, 474, 192195.

(25) Shi, L.; Gruenbaum, S. M.; Skinner, J. L. Interpretation of IR and Raman Line Shapes for $\mathrm{H}_{2} \mathrm{O}$ and $\mathrm{D}_{2} \mathrm{O}$ Ice Ih. J. Phys. Chem. B 2012, 116, 13821-13830.

(26) Duplan, J. C.; Mahi, L.; Brunet, J. L. NMR determination of the equilibrium constant for the liquid $\mathrm{H}_{2} \mathrm{O}-\mathrm{D}_{2} \mathrm{O}$ mixture. Chem. Phys. Lett. 2005, 413, 400-403.

(27) Nagata, Y.; Hasegawa, T.; Backus, E. H. G.; Usui, K.; Yoshimune, S.; Ohto, T.; Bonn, M. The surface roughness, but not the water molecular orientation varies with temperature at the waterair interface. Phys. Chem. Chem. Phys. 2015, 17, 23559-23564.

(28) Wang, H.-F.; Gan, W.; Lu, R.; Rao, Y.; Wu, B.-H. Quantitative spectral and orientational analysis in surface sum frequency generation vibrational spectroscopy (SFG-VS). Int. Rev. Phys. Chem. 2005, 24, $191-256$.

(29) Nihonyanagi, S.; Ishiyama, T.; Lee, T.-k.; Yamaguchi, S.; Bonn, M.; Morita, A.; Tahara, T. Unified Molecular View of the Air/Water Interface Based on Experimental and Theoretical $\chi^{(2)}$ Spectra of an Isotopically Diluted Water Surface. J. Am. Chem. Soc. 2011, 133, $16875-16880$

(30) Zhang, Z.; Piatkowski, L.; Bakker, H. J.; Bonn, M. Ultrafast vibrational energy transfer at the water/air interface revealed by twodimensional surface vibrational spectroscopy. Nat. Chem. 2011, 3, 888-893.

(31) van der Post, S. T.; Hsieh, C.-S.; Okuno, M.; Nagata, Y.; Bakker, H. J.; Bonn, M.; Hunger, J. Strong frequency dependence of vibrational relaxation in bulk and surface water reveals sub-picosecond structural heterogeneity. Nat. Commun. 2015, 6, 8384.

(32) Auer, B. M.; Skinner, J. L. Vibrational Sum-Frequency Spectroscopy of the Water Liquid/Vapor Interface. J. Phys. Chem. B 2009, 113, 4125-4130.

(33) Smit, W. J.; Tang, F.; Nagata, Y.; Sánchez, M. A.; Hasegawa, T.; Backus, E. H. G.; Bonn, M.; Bakker, H. J. Observation and Identification of a New $\mathrm{OH}$ Stretch Vibrational Band at the Surface of Ice. J. Phys. Chem. Lett. 2017, 8, 3656-3660.

(34) Nojima, Y.; Suzuki, Y.; Takahashi, M.; Yamaguchi, S. Proton Order toward the Surface of Ice Ih Revealed by Heterodyne-Detected Sum Frequency Generation Spectroscopy. J. Phys. Chem. Lett. 2017, 8, 5031-5034.

(35) Otsuki, Y.; Sugimoto, T.; Ishiyama, T.; Morita, A.; Watanabe, K.; Matsumoto, Y. Unveiling subsurface hydrogen-bond structure of hexagonal water ice. Phys. Rev. B: Condens. Matter Mater. Phys. 2017, 96, 115405 .

(36) Groenzin, H.; Li, I.; Buch, V.; Shultz, M. J. The single-crystal, basal face of ice $I_{h}$ investigated with sum frequency generation. J. Chem. Phys. 2007, 127, 214502.

(37) Smit, W. J.; Bakker, H. J. The Surface of Ice is Like Supercooled Liquid Water. Angew. Chem., Int. Ed. 2017, 56, 15540-15544.

(38) Ishiyama, T.; Morita, A. A direct evidence of vibrationally delocalized response at ice surface. J. Chem. Phys. 2014, 141, 18 C503.

(39) Bertie, J. E.; Ahmed, M. K.; Eysel, H. H. Infrared Intensities of Liquids. 5. Optical and Dielectric Constants, Integrated Intensities, and Dipole Moment Derivatives of $\mathrm{H}_{2} \mathrm{O}$ and $\mathrm{D}_{2} \mathrm{O}$ at $22^{\circ} \mathrm{C}$. J. Phys. Chem. 1989, 93, 2210-2218.

(40) Li, F.; Skinner, J. L. Infrared and Raman line shapes for ice Ih. II. $\mathrm{H}_{2} \mathrm{O}$ and $\mathrm{D}_{2} \mathrm{O}$. J. Chem. Phys. 2010, 133, 244504. 


\title{
Supporting Information for
}

\section{“Reduced Near-Resonant Vibrational Coupling at the Surfaces of Liquid Water and Ice"}

\author{
Wilbert J. Smit, ${ }^{* \dagger}, \uparrow$ Jan Versluis, ${ }^{\dagger}$ Ellen H. G. Backus, ${ }^{\ddagger}$ Mischa Bonn, ${ }^{\ddagger}$ and \\ Huib J. Bakker ${ }^{\dagger}$ \\ †AMOLF, Science Park 104, 1098 XG Amsterdam, The Netherlands \\ †Max Planck Institute for Polymer Research, Ackermannweg 10, 55128 Mainz, Germany \\ ฯESPCI Paris, PSL Research University, UMR CBI 8231, 10 rue Vauquelin, 75005 Paris, \\ France \\ E-mail: smit@amolf.nl
}




\section{Sample Preparation}

Water samples of various isotopic mixtures are prepared by mixing ultrapure water $\mathrm{H}_{2} \mathrm{O}$ (with a resistivity of $18.2 \mathrm{M} \Omega \mathrm{cm}$ at $298 \mathrm{~K}$ ) with $\mathrm{D}_{2} \mathrm{O}(>99.9 \% \mathrm{D}$, Cambridge Isotope). The liquid water samples are measured in a teflon cup. The production and handling of the monocrystalline $\mathrm{H}_{2} \mathrm{O}$ ice sample are detailed in Ref. ${ }^{\mathrm{S} 1}$ For the production of the $\mathrm{H}_{2} \mathrm{O}: 3 \mathrm{D}_{2} \mathrm{O}$ ice sample an equivalent procedure has been followed with an $\mathrm{H}_{2} \mathrm{O}: 3 \mathrm{D}_{2} \mathrm{O}$ melt maintained at $276.5 \mathrm{~K}$ and a monocrystalline $\mathrm{D}_{2} \mathrm{O}$ seed instead of $\mathrm{H}_{2} \mathrm{O}$.

\section{Heterodyne-Detected SFG Setup and Analysis}

The experimental setup consists of an amplified Ti:sapphire laser system (Coherent Legend) operating at a repetition rate of $1 \mathrm{kHz}$. Infrared pulses are generated by optical parametric amplification and subsequent difference-frequency mixing. The broadband infrared pulses are tuned to a central wavelength of $3.0 \mu \mathrm{m}\left(3300 \mathrm{~cm}^{-1}\right)$ with a full width at half maximum of $550 \mathrm{~nm}\left(600 \mathrm{~cm}^{-1}\right)$ and a pulse energy of $5 \mu \mathrm{J}$. The visible pulse has a central wavelength of $798 \mathrm{~nm}$. The bandwidth of the visible pulse determines the spectral resolution of the SFG spectra. The visible pulse is spectrally narrowed with an etalon to a full width at half maximum of $0.9 \mathrm{~nm}\left(15 \mathrm{~cm}^{-1}\right)$ with an energy of $15 \mu \mathrm{J}$. The visible and infrared pulses are first spatially overlapped on a gold surface to generate broadband sum-frequency light which is used as the local oscillator. Next the visible and infrared pulses are refocused by a concave mirror onto the sample surface under an angle of incidence of $44^{\circ}$ and $39^{\circ}$, respectively. The sum-frequency signal coming from the local oscillator is delayed by a delay plate and creates an interference pattern with the sum-frequency signal generated at the sample, which is recorded by a CCD camera. The signal is extracted by Fourier filtering ${ }^{\mathrm{S} 2}$ and divided by a reference spectrum obtained by replacing the sample by a quartz crystal.

The experiments are performed using s-polarized sum-frequency, s-polarized visible, and $p$-polarized infrared light (i.e. ssp-polarization configuration). The Fresnel factors ${ }^{\mathrm{S} 3}$ are 
calculated using literature values for the optical constants of water, ${ }^{\mathrm{S} 4, \mathrm{~S} 5}$ ice, ${ }^{\mathrm{S} 6}$ and quartz. ${ }^{\mathrm{S} 7}$ The dielectric constant of liquid $\mathrm{HDO}: \mathrm{D}_{2} \mathrm{O}$ is approximated by taking the average of the dielectric constants of $\mathrm{H}_{2} \mathrm{O}$ and $\mathrm{D}_{2} \mathrm{O}$, weighted by their relative concentrations. In the calculation of the dielectric constant of HDO: $\mathrm{D}_{2} \mathrm{O}$ ice, the dielectric constant of $\mathrm{D}_{2} \mathrm{O}$ ice is calculated by frequency shifting the optical constants of $\mathrm{H}_{2} \mathrm{O}$ ice by a factor of $1 / 1.35$. This simplification is justified because $\mathrm{D}_{2} \mathrm{O}$ has no strong vibrational resonances above $2900 \mathrm{~cm}^{-1}$. We verified the validity of our approximation for the liquid phase, for which literature values of the optical constants of both $\mathrm{H}_{2} \mathrm{O}$ and $\mathrm{D}_{2} \mathrm{O}$ are available. ${ }^{\mathrm{S} 4, \mathrm{~S} 5}$ The effective refractive index of the interfacial layers has been calculated using a slab model. ${ }^{\text {S8 }}$ We take the effective $\chi^{(2)}$ value of quartz to be $3 \cdot 10^{-20} \mathrm{~m}^{2} \mathrm{~V}^{-1}$ for our experimental configuration, based on a bulk response of $8 \cdot 10^{-13} \mathrm{~m} \mathrm{~V}^{-1}$. S9

In heterodyne-detected SFG it is essential that the sample and reference are measured at the same height, as a difference in height will lead to a phase shift in the recorded $\chi^{(2)}$ spectrum. The height of the reference and sample is set to a precision of $10 \mu \mathrm{m}$, as determined by the height of the sum-frequency signal on the CCD camera. Evaporation of the liquid water sample during the typical acquisition time of $300 \mathrm{~s}$ is a potential threat to an accurate determination of the phase. ${ }^{\mathrm{S} 10}$ We account for this by setting the initial height of the sample slightly higher, i.e. by half the height loss due to evaporation. This procedure leads to an overall phase uncertainty of the acquired $\chi^{(2)}$ of $\sim 10^{\circ}$. At low isotopic concentrations, the $\operatorname{Im} \chi^{(2)}$ value is very sensitive to the correct reference phase, due to a large non-resonant background contribution. Therefore, we phase-shift the spectra within the phase accuracy to an average $\operatorname{Im} \chi^{(2)}$ of zero between $2850-3000 \mathrm{~cm}^{-1}$ thereby following previous work. ${ }^{\text {S11-S13 }}$ The mean applied phase shift is $6^{\circ}$. After applying the phase correction for each measured spectrum, we average 10-20 spectra for each isotopic concentration.

The SFG measurements on ice are carried out using the temperature-controlled cell previously described in Ref. ${ }^{\mathrm{S} 1}$ The sample cell is moved in the horizontal plane by two motorized actuators to prevent the accumulation of heat and damage of the ice crystal surface. The 
$\chi^{(2)}$ spectra of ice are phase-corrected to an average $\operatorname{Im} \chi^{(2)}$ of zero between $3800-3850 \mathrm{~cm}^{-1}$. Subsequently, 9 spectra for $\mathrm{H}_{2} \mathrm{O}$ ice and 4 spectra for isotopically diluted ice are averaged.

\section{Bulk Infrared and Raman Spectra}

Infrared absorption spectra $\left(\alpha_{\mathrm{IR}}\right)$ are obtained with a PerkinElmer 881 double-beam IR spectrometer. Raman spectra ( $\left.I_{\text {Raman }}\right)$ are taken using a Bruker SENTERRA Raman microscope by excitation by a $532 \mathrm{~nm}$ laser with a power of $10 \mathrm{~mW}$ in an unpolarized manner. The sample is prepared by pressing a water droplet between two windows and is placed in a temperature-controlled cryostat.

\section{Analysis of the Spectra}

In order to quantify spectral changes upon isotope dilution, we numerically determine the full width at half maximum (FWHM) and the first moment of the spectral response $\left(\int \mathrm{d} \omega \omega I(\omega) / \int \mathrm{d} \omega I(\omega)\right)$. The latter depends on the chosen integration window. For the HD-SFG spectra, we restrict the integration domain to the hydrogen-bonded region with negative $\operatorname{Im} \chi^{(2)}$ amplitude, i.e. $3000-3570 \mathrm{~cm}^{-1}$ for liquid water and $3140-3500 \mathrm{~cm}^{-1}$ for ice. For the square-root product of the infrared and Raman spectra, we use the spectral domain 2975-3800 $\mathrm{cm}^{-1}$. We find that the differences in spectral moment between neat $\mathrm{H}_{2} \mathrm{O}$ and $\mathrm{H}_{2} \mathrm{O}: 3 \mathrm{D}_{2} \mathrm{O}$ are not very much dependent on the exact boundaries chosen. An overview of the obtained values is given in Table S1. 
Table S1: Quantitative comparison of the hydrogen-bonded $\mathrm{OH}$ stretching region of $\mathrm{H}_{2} \mathrm{O}$ and $\mathrm{H}_{2} \mathrm{O}: 3 \mathrm{D}_{2} \mathrm{O}$ for both the surface (SFG) and bulk $\left(\sqrt{\alpha_{\mathrm{IR}} \cdot I_{\text {Raman }}}\right)$ spectra. First moments of the spectral response and full width at half maxima are given in $\mathrm{cm}^{-1}$.

\begin{tabular}{lllcc}
\hline \hline & & First moment & FWHM \\
\hline \multirow{3}{*}{ Liquid $(295 \mathrm{~K})$} & \multirow{2}{*}{ Surface } & $\mathrm{H}_{2} \mathrm{O}: 3 \mathrm{D}_{2} \mathrm{O}$ & 3391 & 208 \\
& & $\mathrm{H}_{2} \mathrm{O}$ & 3372 & 269 \\
\cline { 2 - 5 } & \multirow{2}{*}{ Bulk } & $\mathrm{H}_{2} \mathrm{O}: 3 \mathrm{D}_{2} \mathrm{O}$ & 3395 & 306 \\
& & $\mathrm{H}_{2} \mathrm{O}$ & 3361 & 413 \\
\hline \multirow{2}{*}{ Ice $(245 \mathrm{~K})$} & \multirow{2}{*}{ Surface } & $\mathrm{H}_{2} \mathrm{O}: 3 \mathrm{D}_{2} \mathrm{O}$ & 3312 & 91 \\
& & $\mathrm{H}_{2} \mathrm{O}$ & 3279 & 168 \\
\cline { 2 - 5 } & \multirow{2}{*}{ Bulk } & $\mathrm{H}_{2} \mathrm{O}: 3 \mathrm{D}_{2} \mathrm{O}$ & 3309 & 170 \\
& & $\mathrm{H}_{2} \mathrm{O}$ & 3246 & 357 \\
\hline \hline
\end{tabular}




\section{References}

[S1] Smit, W. J.; Tang, F.; Nagata, Y.; Sánchez, M. A.; Hasegawa, T.; Backus, E. H. G.; Bonn, M.; Bakker, H. J. Observation and Identification of a New OH Stretch Vibrational Band at the Surface of Ice. J. Phys. Chem. Lett. 2017, 8, 3656-3660.

[S2] Nihonyanagi, S.; Yamaguchi, S.; Tahara, T. Direct evidence for orientational flipflop of water molecules at charged interfaces: A heterodyne-detected vibrational sum frequency generation study. J. Chem. Phys. 2009, 130, 204704.

[S3] Wang, H.-F.; Gan, W.; Lu, R.; Rao, Y.; Wu, B.-H. Quantitative spectral and orientational analysis in surface sum frequency generation vibrational spectroscopy (SFG-VS). Int. Rev. Phys. Chem. 2005, 24, 191-256.

[S4] Querry, M. R.; Wieliczka, D. M.; Segelstein, D. J. In Handbook of Optical Constants of Solids II; Palik, E. D., Ed.; Academic Press, 1991; pp 1059-1077.

[S5] Max, J.-J.; Chapados, C. Isotope effects in liquid water by infrared spectroscopy. III. $\mathrm{H}_{2} \mathrm{O}$ and $\mathrm{D}_{2} \mathrm{O}$ spectra from 6000 to $0 \mathrm{~cm}^{-1}$. J. Chem. Phys. 2009, 131, 184505.

[S6] Warren, S. G.; Brandt, R. E. Optical constants of ice from the ultraviolet to the microwave: A revised compilation. J. Geophys. Res. 2008, 113, D14220.

[S7] Malitson, I. H. Interspecimen Comparison of the Refractive Index of Fused Silica. J. Opt. Soc. Am. 1965, 55, 1205-1209.

[S8] Zhuang, X.; Miranda, P. B.; Kim, D.; Shen, Y. R. Mapping molecular orientation and conformation at interfaces by surface nonlinear optics. Phys. Rev. B 1999, 59 , $12632-12640$.

[S9] Wei, X.; Hong, S.-C.; Zhuang, X.; Goto, T.; Shen, Y. R. Nonlinear optical studies of liquid crystal alignment on a rubbed polyvinyl alcohol surface. Phys. Rev. E 2000, 62, 5160-5172. 
[S10] Nagata, Y.; Hasegawa, T.; Backus, E. H. G.; Usui, K.; Yoshimune, S.; Ohto, T.; Bonn, M. The surface roughness, but not the water molecular orientation varies with temperature at the water-air interface. Phys. Chem. Chem. Phys. 2015, 17, 2355923564.

[S11] Yamaguchi, S. Development of single-channel heterodyne-detected sum frequency generation spectroscopy and its application to the water/vapor interface. J. Chem. Phys. 2015, 143, 034202 .

[S12] Nihonyanagi, S.; Kusaka, R.; Inoue, K.-I.; Adhikari, A.; Yamaguchi, S.; Tahara, T. Accurate determination of complex $\chi^{(2)}$ spectrum of the air/water interface. J. Chem. Phys. 2015, 143, 124707.

[S13] Stiopkin, I. V.; Weeraman, C.; Pieniazek, P. A.; Shalhout, F. Y.; Skinner, J. L.; Benderskii, A. V. Hydrogen bonding at the water surface revealed by isotopic dilution spectroscopy. Nature 2011, 474, 192-195. 